\title{
COLAB: Módulo LTE de Sensoriamento Colaborativo e Radio Cognitivo para o Simulador de Redes ns-3
}

\author{
Gabriel C. Ferreira ${ }^{1}$, Priscila Solís ${ }^{1}$, Marcos F. Caetano ${ }^{1}$, \\ Marcos V. Lamar ${ }^{1}$, Eduardo A. P. Alchieri ${ }^{1}$, Johanna Vartiainen ${ }^{2}$, \\ Heikki Karvonen ${ }^{2}$
}

${ }^{1}$ Departamento de Ciência da Computação - Universidade de Brasília (UnB)
Caixa Postal 4466 CEP 70910-900 - Brasília - DF - Brasil
gabrielcarvfer@aluno.unb.br, \{pris, mfcaetano, lamar, alchieri\}@unb.br

${ }^{2}$ Centre for Wireless Communications - University of Oulu, Oulu, Finland

\{johanna.vartiainen, heikki.karvonen\}@oulu.fi

\begin{abstract}
The exponential growth of wireless devices connected to the Internet and the limitation of the available electromagnetic spectrum requires the optimization of resource scheduling and frequency reuse to provide a better quality of service. New applications from the evolution of wireless networks, especially the $5 G$ network, consider the use of cognitive radios and the sensing of the spectrum as fundamental elements in its development. The advance and development of new technologies depends on simulation environments that facilitate and integrate tools to evaluate the various applications and scenarios of $5 G$ networks. This article presents a collaborative sensing module and a cognitive resource scheduler for the LS module of the ns-3 network simulator.
\end{abstract}

Resumo. O crescimento exponencial de dispositivos sem fio conectados à internet e a limitação do espectro electromagnético disponível requer a otimização do escalonamento de recursos e reúso de frequências para prover uma melhor qualidade de serviço. As novas aplicações oriundas da evolução das redes sem fio, principalmente a rede $5 G$, consideram o uso de rádios cognitivos e o sensoriamento do espectro como elementos fundamentais no seu desenvolvimento. $O$ avanço e desenvolvimento de novas tecnologias depende de ambientes de simulação que facilitem e integrem ferramentas para avaliar as diversas aplicações e cenários das redes 5G. Este artigo apresenta um módulo de sensoriamento colaborativo e um escalonador de recursos cognitivo para o módulo LTE do simulador de rede ns-3.

\section{Introdução}

A rápida expansão de dispositivos com conectividade sem fio, aliada a novos serviços na internet, geram forte demanda por espectro para transmissão. Para garantir níveis de qualidade de serviço apropriados aos diferentes tipos de aplicações é necessário o aumento da largura de banda e também o aumento da eficiência no uso do espectro disponível. Espera-se que essas necessidades deverão ser atendidas na próxima geração de redes sem fio, ou seja, a quinta geração (5G) que planeja fornecer uma capacidade aumentada em 1.000 vezes, uma taxa de dados 10-100 vezes maior e 10-100 vezes mais dispositivos conectados, em comparação com as atuais redes sem fio $4 \mathrm{G}$. 
Hoje em dia o espectro de radiofrequência está quase totalmente atribuído às diferentes operadoras licenciadas. Para permitir um melhor uso dos recursos de espectro limitado e otimizar a sua utilização são usadas várias tecnologias de multiplexação tais como Acesso Múltiplo por Divisão de Freqüência (FDMA em inglês), Acesso Múltiplo por Divisão de Tempo (TDMA em inglês) e técnicas de Múltiplas Entradas e Múltiplas Saídas (MIMO em inglês). Redes com acesso centralizado (TDMA, CDMA, Ortoghonal FDMA) garantem uma maior eficiência espectral ao evitar o desperdício de recursos com colisões, como ocorre no CSMA (Carrier Sense Multiple Access) ou nas requisições assíncronas para acesso ao meio (CSMA/Collision Avoidance). No controle de acesso ao meio (MAC) centralizado, os pontos de acesso indicam às estações associadas o momento e frequência em que podem transmitir e/ou devem receber uma transmissão, conforme a disponibilidade de recursos de rádio e necessidade das estações. Essas técnicas não se mostram capazes de resolver fundamentalmente o problema de escassez de recursos do espectro causado pela política de alocação fixa [Hu et al. 2018].

Vários estudos mostram [Valenta et al. 2009, Beckman et al. 2010, Hu et al. 2018] que o espectro licenciado é em muitos momentos subutilizado. A tecnologia de Rádio Cognitivo (RC) surgiu como uma proposta para resolver o problema de subutilização do espectro e otimizar a alocação de recursos. O espectro de radiofrequência apresenta um conjunto de faixas não contínuas de espectro não utilizados pelos respectivos usuários licenciados chamados de usuários primários (PUs em inglês). O sensoriamento de espectro é o primeiro passo do compartilhamento de espectro e permite que usuários secundários (SUs em inglês) usem de maneira oportunística as faixas de espectro vazias de acordo com sua necessidade e deixem de acessar o canal tão logo o PU retome sua transmissão. Este compartilhamento é chamado de acesso dinâmico ao espectro (DSA em inglês) e permite o uso de espectro ocioso desde que não interfira com o PU. A correta execução do procedimento permite aumentar a largura de banda disponível para os SUs e é considerada fundamental para o desenvolvimento das redes $5 \mathrm{G}$.

Normalmente, o uso compartilhado do espectro envolve quatro passos: 1) sensoriamento; 2) alocação; 3) acesso e 4) hand-off. Trabalhos recentes de pesquisa [Hu et al. 2018] agrupam as técnicas de sensoriamento do espectro em 3 grandes grupos: a) não colaborativos, onde os SUs detectam o PU e evitam o canal, ideal para uma abordagem de protocolos MAC descentralizados; b) base de dados, em que cada SU envia o resultado do sensoriamento para o ponto de acesso ou estação rádio base, que compara resultado com dados históricos para encontrar faixas disponíveis e c) colaborativo, que combina os resultados de detecção de vários SUs para melhorar a confiabilidade da detecção usando algoritmos de fusão.

Amplamente usado no meio acadêmico, o ns-3 [NSNAM 2019] é um simulador de rede de eventos discretos de código aberto. Comparado a outros simuladores de código aberto, o ns-3 oferece alguns recursos de simulação multi-RAT (Radio Access Technology) e multi-banda, com Wi-Fi, WiGig, LTE (Long Term Evolution) (LTE-A, LAA, LTE-U), entre outros. Existe um interesse de estender as funcionalidades do ns-3 para permitir avaliar as tecnologias que promoverão o desenvolvimento da rede $5 \mathrm{G}$, entre elas, os RC e as técnicas DSA. Existem várias iniciativas nesse sentido, por exemplo o LENA [CTTC 2019], desenvolvido para o LTE no ns-3. Entretanto, no momento em que 
a presente ferramenta foi desenvolvida, não existia ainda uma implementação sólida de algoritmos de sensoriamento colaborativo com foco em redes LTE e 5G.

Com essa motivação, este trabalho apresenta o COLAB, um conjunto de adaptações para o módulo LTE do simulador ns-3 que implementa o sensoriamento colaborativo para rádio cognitivo. A implementação e validação deste módulo para o ns-3 utilizando a pilha LTE serve de base para a simulação de uma rede 3GPP [Sultan 2019], facilitando comparações de resultados e portabilidade de novos protocolos para a pilha do 5G-NR. O algoritmo de sensoriamento permite que a torre de telefonia chamada de evolved NodeB (eNB) estime o comportamento de um PU e aloque recursos de rádio de maneira a evitar colisões. O protótipo do sensoriamento colaborativo foi desenvolvido em $\mathrm{C}++\mathrm{e} u m$ conjunto de ferramentas auxiliares para plotagem de resultados foram implementadas em Python.

As contribuições esperadas do COLAB à área de pesquisa em redes $5 \mathrm{G}$ são as seguintes: a) suporte ao desenvolvimento de novos algoritmos de escalonamento da camada de enlace, com base nas informações do sensoriamento colaborativo; b) suporte ao desenvolvimento de novas técnicas para caracterização do padrão de acesso ao canal do PU, a fim de permitir a utilização oportunística do canal sem causar prejuízos à transmissão primária; c) verificação do impacto dos mecanismos de sensoriamento da camada física nas camadas superiores e nas métricas das diversas aplicações das redes LTE e 5G; d) facilitar um ambiente para o desenvolvimento de algoritmos de detecção de espectro ideal em conjunto com estratégias de alocação de espectro e mecanismos de acesso ao espectro que atendam aos diferentes requisitos dos quatro cenários de aplicação das redes 5G. Este artigo está organizado como segue: a Seção 2 apresenta a especificação da arquitetura usada no ns-3 para implementar o algoritmo de sensoriamento colaborativo e seu uso com rádio cognitivos. A Seção 3 descreve o roteiro de demonstração da ferramenta. A Seção 4 apresenta as conclusões e trabalhos futuros.

\section{Descrição da ferramenta}

\subsection{Arquitetura e funcionalidades}

A implementação da arquitetura para sensoriamento colaborativo adotada no módulo LTE do simulador ns-3 é dispersa pelas múltiplas classes que implementam as diferentes camadas da pilha do protocolo, conforme mostrado na Figura 1. À camada LteSpectrumPhy, que corresponde à parte de transmissão e recepção de sinais, no método de recepção (StartRX) foi acrescida uma parte para integração dos dados recebidos e o encaminhamento para uma função de sensoriamento (Sense). A função StartRxDlCtrl foi modificada para permitir o recebimento de mensagems de controle com resultados do sensoriamento e o seu encaminhamento para a camada PHY do eNB (eNodeB, implementado no LteEnbPhy).

À camada MAC do módulo do UE (User Equipment), foi acrescida uma função para montagem da mensagem de controle com resultados do sensoriamento (SendCognitiveMessageC). Ainda à camada MAC do eNB, foram acrescidas funções para receber mensagens de sensoriamento das diferentes estações (ReceiveCognitiveMessage) e fazer fusão dos resultados (mergeSensingReports). Assim também, a função DoSubframeIndication 


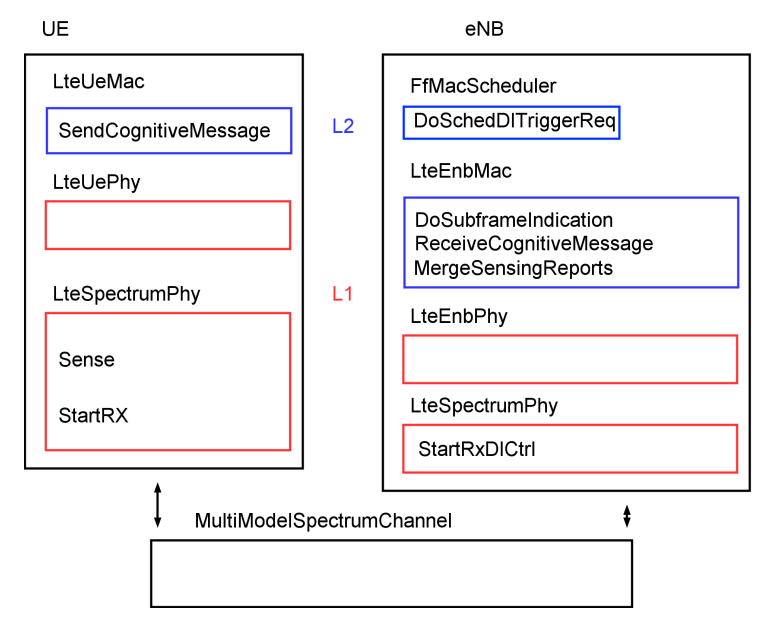

Figura 1. Implementação de pilha de rede LTE com sensoriamento no ns-3.

do LTE foi modificada, para implementar a fusão de resultados antes de executar o escalonamento de recursos. Ainda na camada MAC do eNB, a função DoSchedDlTriggerReq dos escalonadores passou a receber um parâmetro adicional, contendo um bitmap informando os grupos de blocos de recursos (RBGs em inglês) ocupados, prevenindo seu uso pelo escalonador.

\subsection{Funcionamento dos Módulos Desenvolvidos}

A Figura 2 mostra um esquema dos passos do algoritmo desenvolvido no plano de controle para a operação do sensoriamento colaborativo em um canal licenciado. A primeira versão dessa implementação no módulo exemplifica o acesso do usuário primário (PU) ao canal em um dado momento, seguida pela notificação da UE para o eNB sobre a presença do PU, a agregação das notificações pelo eNB e o seu efeito no escalonamento de recursos para prevenir transmissões que interfiram com o PU.

Na camada física (LteSpectrumPhy), está disposta a função de sensoriamento do canal (Sense), que executa o sensoriamento. Dado que o ns-3 não emula a camada física no caso da rede LTE, o algoritmo de sensoriamento do canal utiliza os parâmetros de SINR e uma curva de probabilidades de detecção de usuário primário, gerados externamente em um simulador de camada de enlace. O protótipo de algoritmo de sensoriamento implementado (sensingP rocedure), verifica a relação SINR de cada um dos RBGs, ou a relação entre o SINR médio dos RBGs, e estima as chances de um PU estar transmitindo baseado na curva de probabilidade. A Figura 3 mostra um exemplo de curva de probabilidade de detecção que serve de entrada no ns-3, calculada em um ambiente simulado com o uso de vários pontos de amostragem e parâmetros do canal físico de interesse[5G-Range 2018]. A probabilidade de falso positivo é Pfa $=0.01$. O módulo da camada física dos eNBs (LteEnbPhy) foi modificado para redirecionar mensagens com informações do sensoriamento cognitivo para a camada MAC do eNB associado.

Na camada MAC dos UEs (LteUeMac), se encontra a função de notificação da detecção de PU via canal de controle (SendCognitiveMessageC), ou via canal de dados (SendCognitiveMessage). A notificação contém o número do quadro e sub-quadro, mais um bitmap indicando os RBGs em que a transmissão do PU foi detectada. A camada MAC dos eNBs (LteEnbMac) foi modificada para receber as men- 


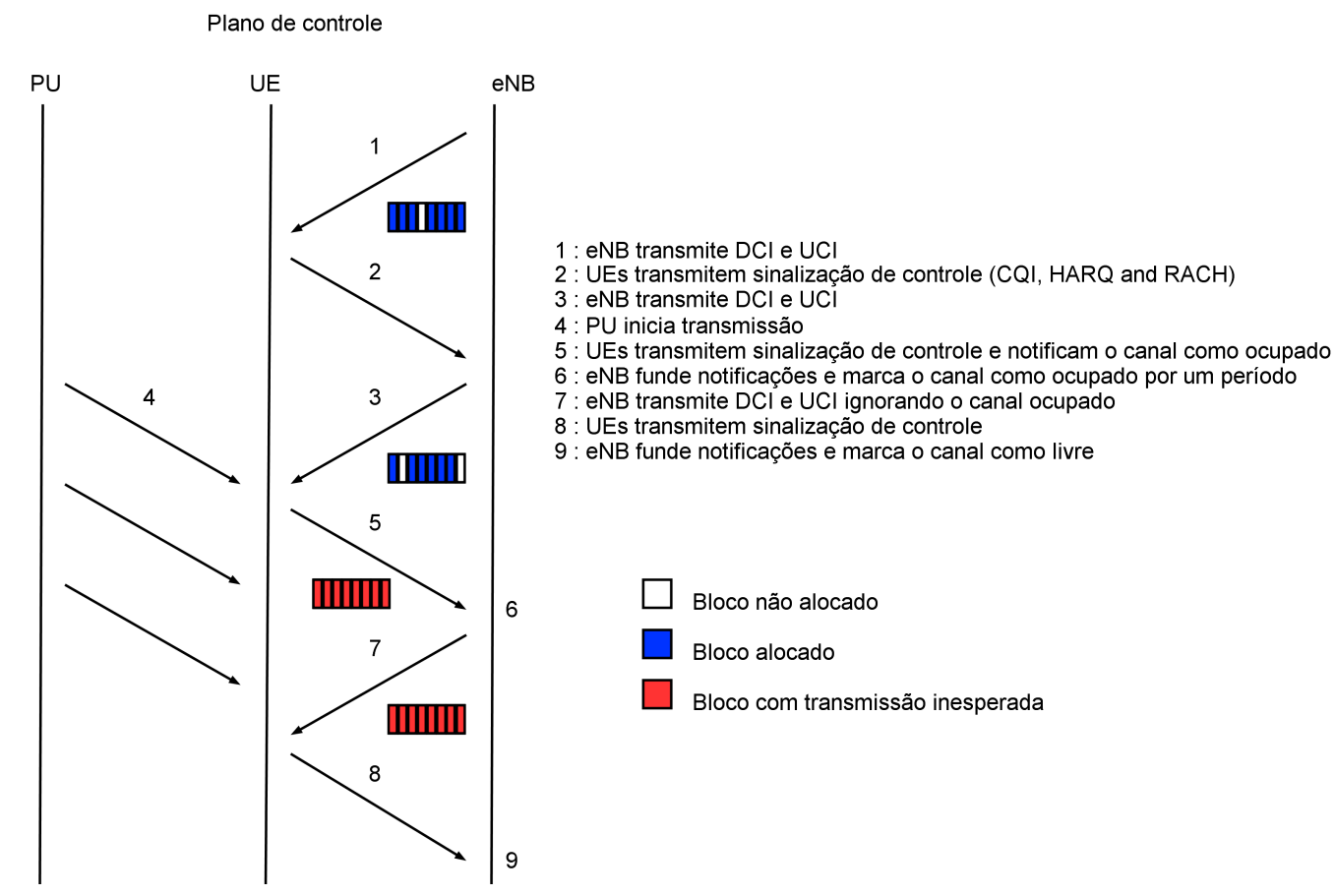

Figura 2. Diagrama do sensoriamento colaborativo

sagens do sensoriamento colaborativo (RecvCognitiveMessage, via canal de dados, ou RecvCognitiveMessageC, via canal de controle), guardando em seguida os resultados dos sensoriamentos dos UEs em registros que facilitam a posterior consulta. Ainda na camada MAC dos eNBs (LteEnbMac), dentro da função de indicação do sub-quadro (DoSubframeIndication) e antes do disparo de cada escalonamento (SchedDlTriggerReq), foi implementado um método que agrega os dados do sensoriamento colaborativo. O resultado dessa agregação é um bitmap de RBGs a serem ignoradas pelo escalonamento, evitando interferir com o usuário primário.

Um exemplo dos resultados da execução da ferramenta é mostrado na Figura 5, utilizando a topologia da Figura 4, que contém 10 dispositivos (UEs) e um eNodeB. O primeiro gráfico da Figura 5 mostra a potência de transmissão medida do PU no canal. No segundo gráfico, é mostrado o resultado do sensoriamento individual dos UEs ao longo da simulação. No terceiro gráfico, é mostrada a relação SINR média dos RBGs para cada um dos UEs ao longo da simulação, visualmente indicando a presença da transmissão do PU. No quarto gráfico, são plotados os resultados do escalonamento, com espaços em branco indicando RBGs livres, azul indicando RBGs escaladas (transmissões de downlink), enquanto RBGs com transmissões do PU são indicadas em vermelho, onde transmissões LTE cessam. Este gráfico reflete diretamente o algoritmo de agregação das informações de sensoriamento colaborativo advindo dos UEs, podendo aumentar ou reduzir o número de falsos positivos e/ou falsos negativos.

\subsection{Aplicações e Casos de uso}

A presente ferramenta pode ser utilizada como um suporte para o desenvolvimento e avaliação dos seguintes casos de uso:

- Novos algoritmos de escalonamento para a camada MAC: algoritmos clássicos 


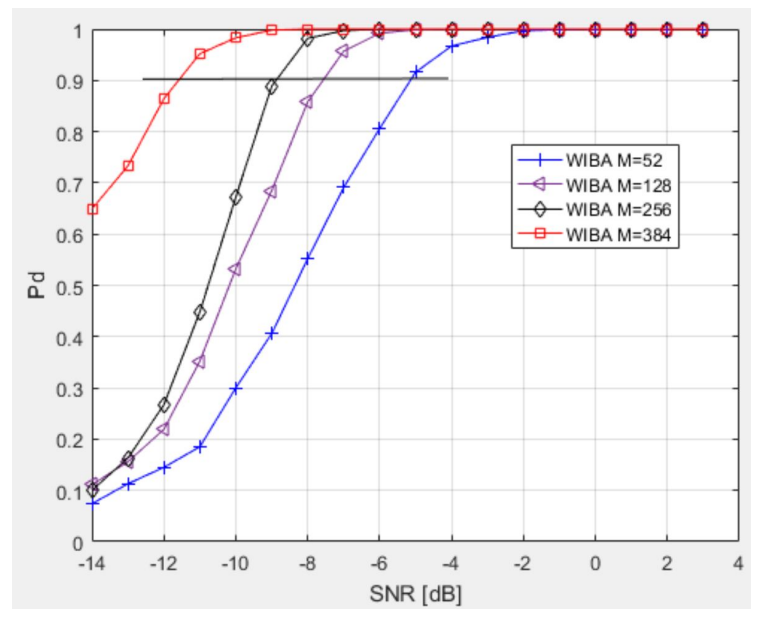

Figura 3. Curvas de probabilidade de detecção do PU vs SNR [5G-Range 2018]

de escalonamento tipicamente assumem que o canal é licenciado e, portanto, o eNB é usuário primário. O sensoriamento colaborativo implementado na ferramenta provê informações suficientes para a modificação e implementação de novos algoritmos que levem em conta a possibilidade de usar oportunisticamente um canal licenciado. A ferramenta assume que o canal de controle fica em banda licenciada e não sofre interferência de terceiros.

- Caracterização do padrão de acesso do PU ao canal e rádio cognitivo: o acesso à whitespaces, é tipicamente regido por bases de dados controlados por agências controladoras do espectro. As bases de dados porém, não contam com comportamento detalhado do padrão de acesso do PU, o que dificulta o reúso do espectro por terceiros. Neste sentido, a ferramenta oferece a possibilidade da implementação de algoritmos de aprendizado para identificar e caracterizar o padrão de acesso do PU, a fim de prever seus acessos e identificar oportunidades de acesso.

- Efeitos colaterais no sistema baseados no extrapolação do sensoriamento real: a ferramenta pode ser utilizada para verificar como diferentes mecanismos de sensoriamento da camada física, seja real ou simulada, afetam a rede como todo. $\mathrm{O}$ método sensingProcedure carrega uma curva de probabilidade da detecção de um PU, que pode ser substituída facilmente; permite a detecção para cada RBG ou no canal como todo, dependendo da necessidade do algoritmo de sensoriamento utilizado; pode ser feito apenas em RBGs não alocados para transmissão, evitando RBG escaladas para downlink (DCIs), uplink (UCIs) e retransmissões HARQ (RAR).

\section{Roteiro de Demonstração}

O código fonte, manual e vídeos estão disponíveis em https: / / gabrielcarvfer. github.io/NS3/COLAB/.

A demonstração da ferramenta se dará em um conjunto de cenários com e sem acesso dinâmico ao espectro, onde uma rede LTE utiliza oportunisticamente um canal licenciado para a transmissão de dados, coexistindo com o PU do canal. O cenário simulado difere do padrão LTE-LAA, onde a rede LTE faz uso de um canal não licenciado utilizando o princípio LBT (Listen-Before-Talk). A detecção de transmissões do 


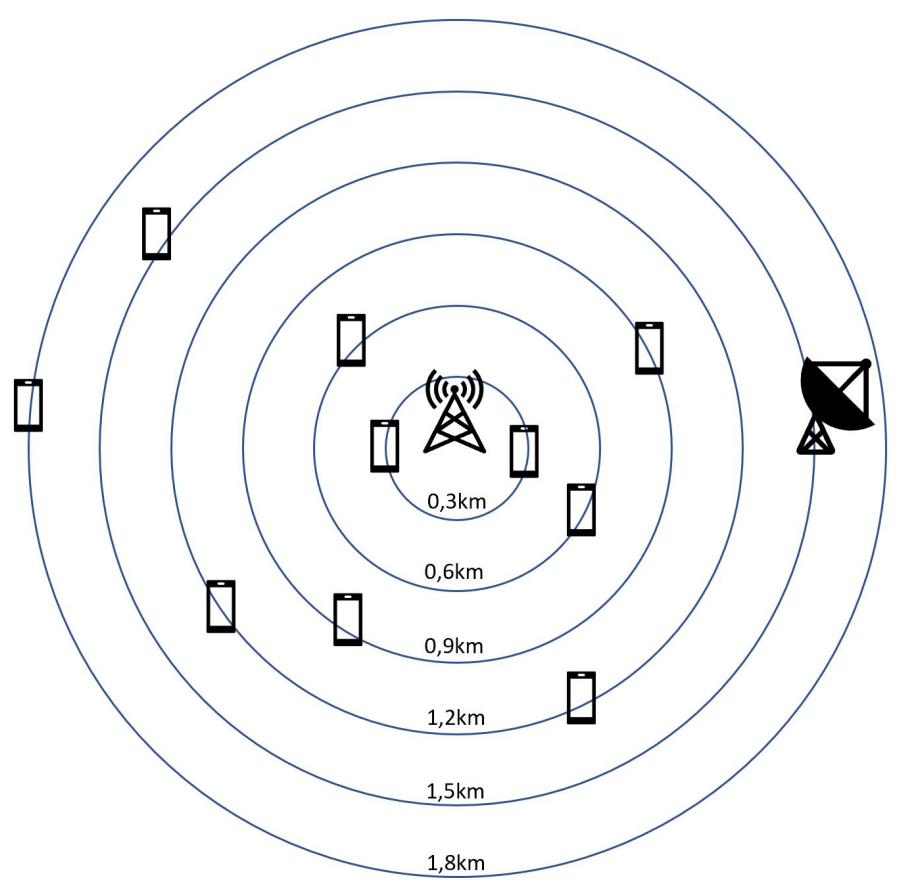

Figura 4. Topologia do cenário de simulação para sensoriamento colaborativo
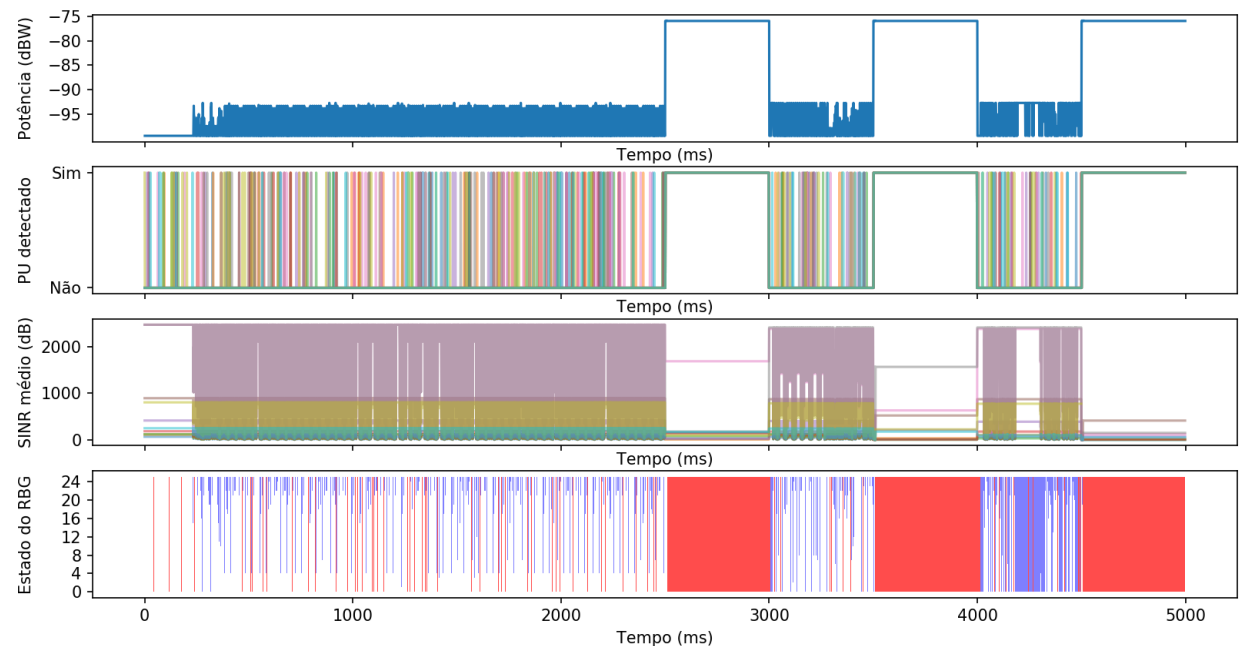

Figura 5. Resultados do sensoriamento colaborativo e reflexo no escalonamento de recursos 
PU através do sensoriamento colaborativo e cessão das transmissões no canal licenciado, prevem e/ou reduzem a geração de interferência que afete o PU. A topologia da rede utilizada nos cenários é a mesma utilizada na Seção 2.2, mostrada na Figura 4. A curva de probabilidade utilizada é a mostrada na Figura 3.

Os cenários exemplificados na demonstração são 04, resultantes das combinações de um canal AWGN (Additive white Gaussian noise) de $20 \mathrm{MHz}$, com ruído de fundo de $-174 \mathrm{dBm} / \mathrm{Hz}$, com e sem a presença do usuário primário, com e sem DSA. As métricas comparadas são latência, vazão, interferência percebida à transmissão do usuário primário (medição de SINR) e taxa de utilização do canal.

\section{Conclusão e trabalhos futuros}

A escassez de recursos de rádio e a demanda por melhor cobertura e qualidade de serviço tornam evidentes a necessidade do desenvolvimento e aprimoramento de tecnologias que permitam o uso oportunístico do espectro. O COLAB, a ferramenta ora apresentada, pode ajudar na pesquisa, desenvolvimento e teste de algoritmos para redes cognitivas e acesso oportunístico integrado à pilha LTE do ns-3. Como trabalhos futuros, serão investigados novos métodos para aprimorar o ciclo cognitivo de sensoriamento, algoritmos de inteligência artificial para predição do comportamento do PU e alocação de RBGs, algoritmos de fusão dos dados de sensoriamento, melhorando os resultados da ferramenta e das redes simuladas.

\section{Agradecimentos}

Este trabalho foi realizado com suporte do projeto 5G-Range (www.5g-range.eu), aprovado na 4a. Chamada EU-BR em TICs, financiada pelo CTIC/RNP/MCTIC.

\section{Referências}

5G-Range (2018). Spectrum sensing to complement databases. Technical report. http://5g-range.eu/wp-content/uploads/2018/04/D4. 2-Spectrum-Sensing-to-Complement-Databases.pdf.

Beckman, R., Channakeshava, K., Huang, F., Vullikanti, V. S. A., Marathe, A., Marathe, M. V., and Pei, G. (2010). Implications of dynamic spectrum access on the efficiency of primary wireless market. In 2010 IEEE Symposium on New Frontiers in Dynamic Spectrum (DySPAN). IEEE.

CTTC (2019). The first release of 5g-lena is available. http://www. cttc.es/ the-first-release-of-5g-lena-is-available/.

$\mathrm{Hu}, \mathrm{F}$., Chen, B., and Zhu, K. (2018). Full spectrum sharing in cognitive radio networks toward 5g: A survey. IEEE Access, 6:15754-15776.

NSNAM (2019). ns-3 network simulator. https : / /www . ns nam. org/.

Sultan, A. (2019). 21.915 release 15. Technical report. https://portal.3gpp. org/desktopmodules/Specifications/SpecificationDetails. aspx?specificationId=3389.

Valenta, V., Fedra, Z., Marsalek, R., Baudoin, G., and Villegas, M. (2009). Towards cognitive radio networks: Spectrum utilization measurements in suburb environment. In 2009 IEEE Radio and Wireless Symposium. IEEE. 